\title{
Application and validation of profile-image method for measuring deformation of tunnel wall
}

\author{
Tai-Tien Wang $^{\mathrm{a}, *}$, Jen-Jer Jaw ${ }^{\mathrm{b}}$, Yo-Ho Chang ${ }^{\mathrm{c}}$, Fu-Shu Jeng ${ }^{\mathrm{b}}$ \\ a Department of Materials and Mineral Resources Engineering, National Taipei University of Technology, Taipei, Taiwan \\ b Department of Civil Engineering, National Taiwan University, Taipei, Taiwan \\ c Institute of Earth Sciences, National Hualien University of Education, Hualien, Taiwan
}

\section{A R T I C L E I N F O}

\section{Article history:}

Received 10 August 2007

Received in revised form 30 April 2008

Accepted 27 May 2008

Available online $\mathrm{xxxx}$

\section{Keywords:}

Safety inspection

Tunnel wall

Deformation

Profile-image method

Laser

\begin{abstract}
A B S T R A C T
The deformation of a tunnel is one of the major phenomena that indicate the safety of a tunnel under operation. Yet, the deformation of tunnel profiles so far cannot be effectively obtained, as confined by the long time required when using conventional geodetic surveying and the insufficient precision provided by other devices. The profile-image method, by which the profile of a tunnel can be determined by laser-lit profile in an image is tested in this study to validate its applicability. The methodology, including superimposing of images, and the results of validations are presented. The results indicate that the profile-image method has a precision of $4 \mathrm{~mm}$ for medium size tunnel. Meanwhile, the factors affecting the precision of measurements are highlighted, including distortion of lens, number of calibration points, types of lasers, and contrast of profile to the background. The influences of these factors are also presented.
\end{abstract}

(c) 2008 Elsevier Ltd. All rights reserved.

\section{Introduction}

\subsection{Background}

Since the deformation of an operating tunnel may serve as a direct indicator regarding the variation of safety, the deformation is of major concern especially when conducting maintenance inspection for such a tunnel. The subsequent deformation of a tunnel after construction inherently is a result of ambient stress changes, which will also affect the state of safety of the tunnel. This deformation can also be used for back analysis to reveal what conditions, e.g. ambient stress changes and existence of cavity, have occurred. Therefore, it is possible that the deformation of tunnel (or its inner lining) can be adopted as a criterion for evaluating the current state of safety, and for providing precaution for upcoming disasters (Japanese Society of Civil Engineers, 2002, 2003; Wang et al., 2005, 2007a). From the viewpoint of maintenance, it is preferable to well quantify the quantities and the pattern of deformation of tunnel lining, and to observe deformation on a regular basis.

However, to measure the deformation of a tunnel currently is not a common practice owing to the time and cost required by this operation. Especially, for an operating tunnel, e.g. a highway tun-

\footnotetext{
* Corresponding author. Tel.: +886 22771 2171x2773; fax: +886 227317185

E-mail address: ttwang@ntut.edu.tw (T.-T. Wang).
}

nel, the traffic must be stopped when conducting surveying profiles so that the time allowed for survey is really limited.

Many methods have been developed to measure the profiles of a tunnel, and the deformation of a profile can be accordingly obtained by comparing the previous profile and the current profile. Conventionally, a profile can be precisely yet rather slowly pointby-point measured by geodetic devices. Laser devices capable of automatically scanning an entire section of a tunnel, resulting in tens of thousands of points with their spatial coordinates, the socalled "cloud of points", are also available (Langer et al., 2000; Wang et al., 2007b). The third method, direct laser beam measuring the relative position of a point at tunnel wall to the laser device has been also utilized. If the trace of such a laser device is recorded, the spatial coordinate of each measured point can then be determined. By doing this, the profile can be accordingly obtained.

However, conventional geodetic survey is rather time consuming and thus is difficult to meet the requirement of limited time allowed. On the other hand, both the automatic laser scanning and the direct measurement using laser beam can be conducted rather fast but require further validation on the precision of the measurements.

Further strategies have been adopted by researchers to cope with these difficulties (Lichti et al., 2000; Boehler et al., 2001; Boehler and Marbs, 2002). One popular approach is the laser range technology on the basis of traveling time of laser beam, which usually integrates a rotating mirror with a laser ranger to get all-round angle of data. The accuracy of this approach depends on the errors 
of measured distance and gauged angle, and is usually limited to $10 \mathrm{~mm}$ or worse (Chao, 2006; Chiu, 2007). Another possibility is to use the stereo images technology based on the triangulation, which analyzes stereo pairs taken from various positions to obtain the $3 \mathrm{D}$ coordinates of lining surface. Its precision is restricted by the resolution of the images, the geometry of intersected rays of conjugated points, and the orientation parameters of the images. In general, the configuration of stereo images is rather troublesome due to the space limitation in a tunnel.

Finally, a third strategy is also founded on the triangulation, but only a single image is used. The line connecting the illuminating laser and the camera is adopted as a base, which correlates the line connecting the object and the camera with the angle in-betweens to form a triangulation. An intelligent alternative of the third strategy is to specify some points with known coordinates, which are referred as "calibration points" or "control points", within an image so that these points can be used to determine the coordinates of any particular points within the image. The coordinate of these calibration points is precisely determined using geodetic device before an image is taken. This method is referred as "profile-image method" herein. This method has been successfully used in small scale laboratory tests for flow surface measurement (Capart et al., 2002; Douxchamps et al., 2005).

As such, it is of interest to extend this method to measure the profile of any section of a tunnel. Many questions may follow this extension: How can this method be extended to measure the profile of a tunnel? What is the precision? How fast is the measuring operation? etc. The study also aims at finding the answers for these questions.

\subsection{Basics of profile-image method}

As depicted in Fig. 1, the fundamental concept of imaging method is to find coordinates of any specified points from 6 points with known coordinates. All the specified points and the 6 points with known coordinates, referred as "calibration points" herein $(\mathrm{Ni}$, 2005), should be first taken into one image by camera. If the profile of an object is desired, a line laser can be used to beam the surface of the object so that the connection of all the illuminated points on the surface in fact constitutes a profile. As shown in Fig. 1, the camera is located at point $P$, the coordinates of points alone line (or profile) $S$ are to be determined, and these specified points are included in the image.

After the specified points are included in one image, they can be readily related to the local coordinate of the image, as shown by the image in Fig. 1. The next task is to find the transformation relationship of the global 3D coordinates and the local 2D coordinates. This transformation of coordinates can be found using perspective projection method (Faugeras and Luong, 2001). The following notations are adopted throughout this paper: lower-case letters denote $2 \mathrm{D}$ coordinates measured in the image taken; upper-case letters denote the global 3D coordinates in real space. For both cases, vectors are indicated in bold face.

Fig. 1 illustrates that the position vector $\boldsymbol{S}$ of an arbitrary point $S$ in global coordinate system can be described by the position vector $\boldsymbol{P}$ of projection centre and the ray direction $\boldsymbol{q}$, respectively. The 3D coordinates $\boldsymbol{S}$ are related to the 2D coordinates $\boldsymbol{q}$ as

$\boldsymbol{S}=\boldsymbol{P}+\lambda \boldsymbol{q}$

where $\lambda$ is a scalar parameter. The vector $\boldsymbol{q}$ can be directly measured from the image, at any specified point on $s$, and indicated as $\left(x^{s}, y^{s}\right)$.

Given a line parallel to $X-Z$ plane, the global coordinates of $S=\left(X^{S}, Y^{S}, Z^{S}\right)$ can be determined by projection centre $P=\left(X^{P}, Y^{P}, Z^{P}\right)$ and the image position $\left(x^{S}, y^{S}\right)$. Accordingly, the following relation can be established:

$$
\left(X^{S}, Y^{S}, Z^{S}\right)^{\mathrm{T}}=\left(X^{P}, Y^{P}, Z^{P}\right)^{\mathrm{T}}+\lambda \boldsymbol{M}\left(x^{S}, y^{S}, 1\right)^{\mathrm{T}}
$$

where the superscript $\mathrm{T}$ represents transpose of raw vectors and $\boldsymbol{M}$ is a $3 \times 3$ transformation matrix corresponding to the orientation and position of the image.

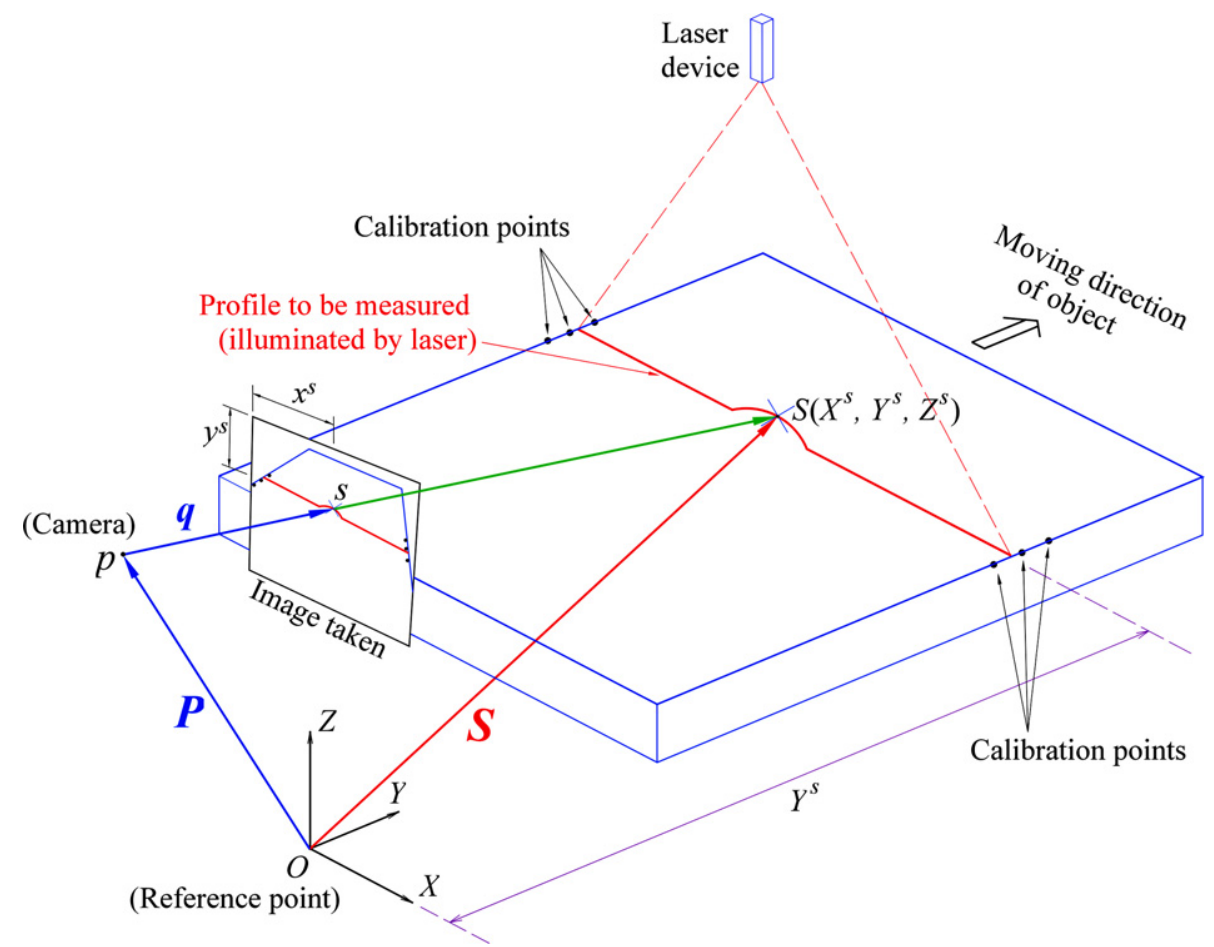

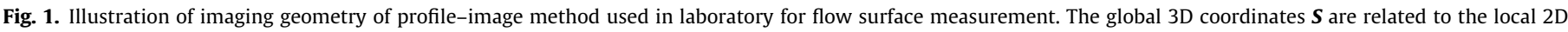

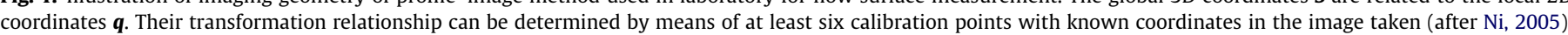


By rearranging Eq. (2), we have

$\left(\lambda x^{S}, \lambda y^{S}, \lambda\right)^{\mathrm{T}}=\boldsymbol{A}\left(X^{S}, Y^{S}, Z^{S}\right)^{\mathrm{T}}+\boldsymbol{B}$

where $\boldsymbol{A}$ is the inverse matrices of $\boldsymbol{M}$, and $\boldsymbol{B}=-\boldsymbol{M}^{-1}\left(X^{P}, Y^{P}, Z^{P}\right)^{\mathrm{T}}$. As a result, there are 11 unknown coefficients in matrix $\boldsymbol{A}$ and $\boldsymbol{B}$, and thus 11 equations are required to determine them. On the other hand, each calibration point provides two equations, in $x$-direction and in $y$-direction, for solving those unknowns (Capart et al., 2002). Accordingly, the transformation would be accomplished if no less than six calibration points are provided and included in the image. If more than six calibration points are provided within an image, then the precision can be further improved, taking advantages of the special information provided by these surplus calibration points.

With reference to Fig. 1, the points, forming a profile $S$, to be surveyed are highlighted by a line laser and acquired by a digital camera. A laser light-pot, although it may look fairly small in the taken image, in fact is constituted by more than one pixel. The next task, referred as "particle identification", is to identify a representative pixel, which best represents the position of the laser spot. Therefore, image processing method should be undertaken to pinpoint position of a laser spot from the image in order to precisely retrieve its $2 \mathrm{D}$ location, which ensures a better 3D coordinates to be converted.

The techniques regarding image processing and the particle identification have been discussed in detail by Jähne (1995), Capart et al. (2002) and Spinewine et al. (2003). In general, three main steps are involved: (1) to increase the contrast of the laser lightspots by assigning different degrees of grey-scale colors to the spots and to the background; (2) to eliminate noises by applying low-pass and high-pass filters; and (3) to identify the representative pixel, which is brightest in the spot, for each individual laser point. As a result, the 2D coordinates of laser points, measured in laboratory, can be determined with fairly well precision (Veber et al., 1997; Capart et al., 2002; Ni, 2005).

\subsection{Scope of this study}

This work aims to extend the application of the profile-image method, from laboratory scale to field scale, to measure the profile of tunnel wall. By doing this, the profiles of a same section measured at different investigations can be compared and the deformation, if it does occur, can be identified. A few questions follow this extension of application: (1) How is the precision? (2) What are the factors affecting the results of measurement and the degree of influences? and (3) Will the field conditions, e.g. background illumination of a tunnel, affect the precision? On the basis of laboratory-scale image method, some technological transformations have been implemented in order to match the requirement for survey task in real tunnel-scale. Therefore, a series of tests are designated to validate these technological transformations. The precision and the efficiency of field work are the main items to be observed during the validation process. Finally, the factors affecting the results of the profile-image method are discussed.

\section{Framework of research}

The original profile-image method was applied to measure the profile of a movable object so that the line laser was fixed in space and the object moved through the line laser to obtain profiles for different sections, as shown in Fig. 1. As such, the six calibration points and camera are also fixed in space. When extending profile-image method to tunnels, the tunnel cannot move through the line laser and, instead, the entire measuring system must move along the course of tunnel, as shown in Fig. 2. Since at least six cal-

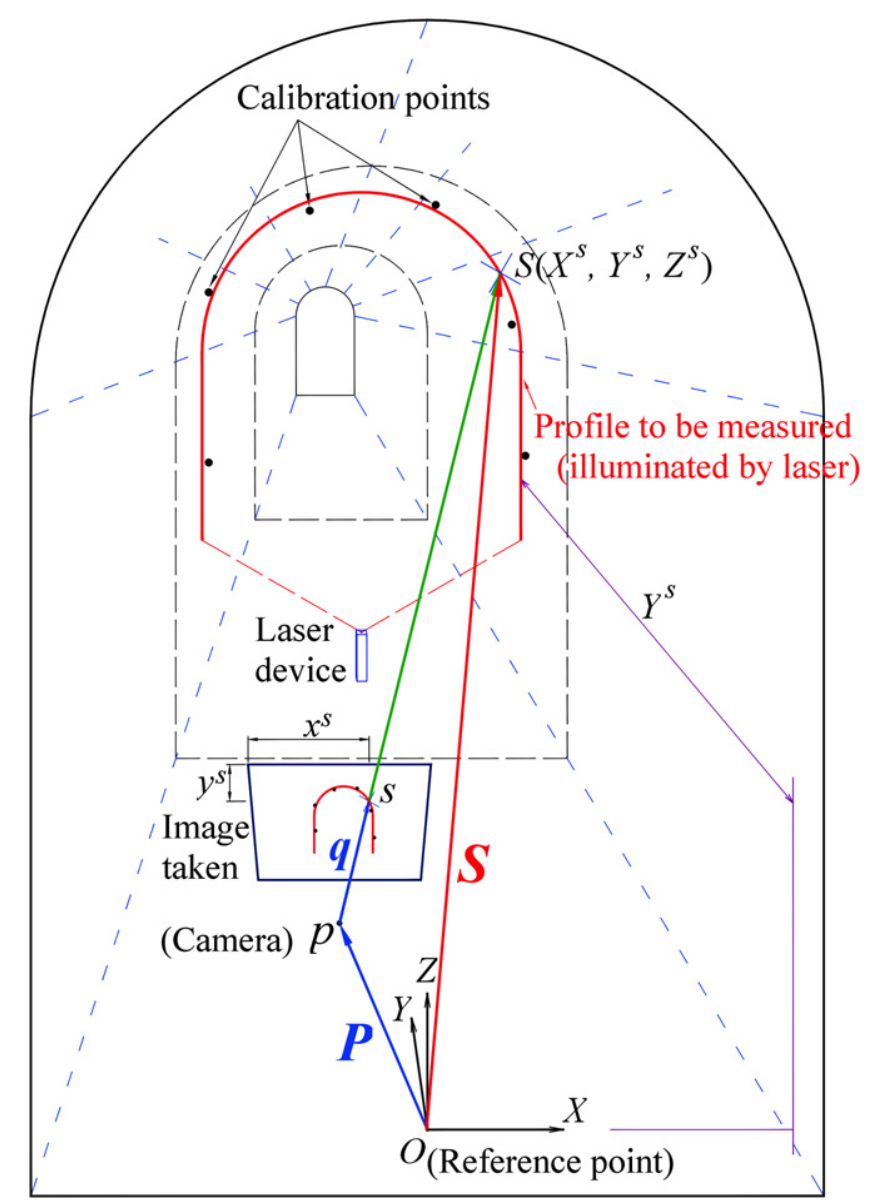

Fig. 2. Illustration of imaging geometry of profile-image method for the survey of tunnel wall. Laser device is used to beam the profile to be measured. The calibration points have to be set up within the tunnel and taken into the image.

ibration points should be included in an image, many calibration points, with their 3D coordinates measured by geodetic device, should be set up within the tunnel. Therefore, the procedures for applying profile-image method to tunnels should be slightly modified from the original method, and are summarized in Fig. 3.

Owing to the nature of profile-image method, the coordinates of the points along a profile are determined by the six calibration points, and the location (or coordinates) of camera is not needed. Therefore, the positions of the camera inside the tunnel are not measured. Remarkably, the planes of the image (the CCD of camera) and the profile should be parallel, as shown in Fig. 2.

The 3D coordinates of the calibration points can be selected to be relative to a fixed point, which is often located at tunnel portal. If the global coordinates of this fixed point, referred as "reference point" herein, are measured, then the global coordinates of all calibration points can also be determined.

The conditions of a tunnel can be different from the ones in the laboratory, for instance, the size for a tunnel survey is much larger than the object measured in laboratory scale. Since the tunnel is much larger, the power of line laser adopted for laboratory measurements is no longer sufficient and a laser device with much stronger power should be employed. On the other hand, the in situ conditions of a tunnel can also be different, e.g. the background illumination. Too strong illumination lowers the contrast of laser line; yet too dim background may prevent correct focusing of the camera.

In the situations that the tunnel is quite large so that the laser power is not sufficient to yield a bright enough profile shown in 


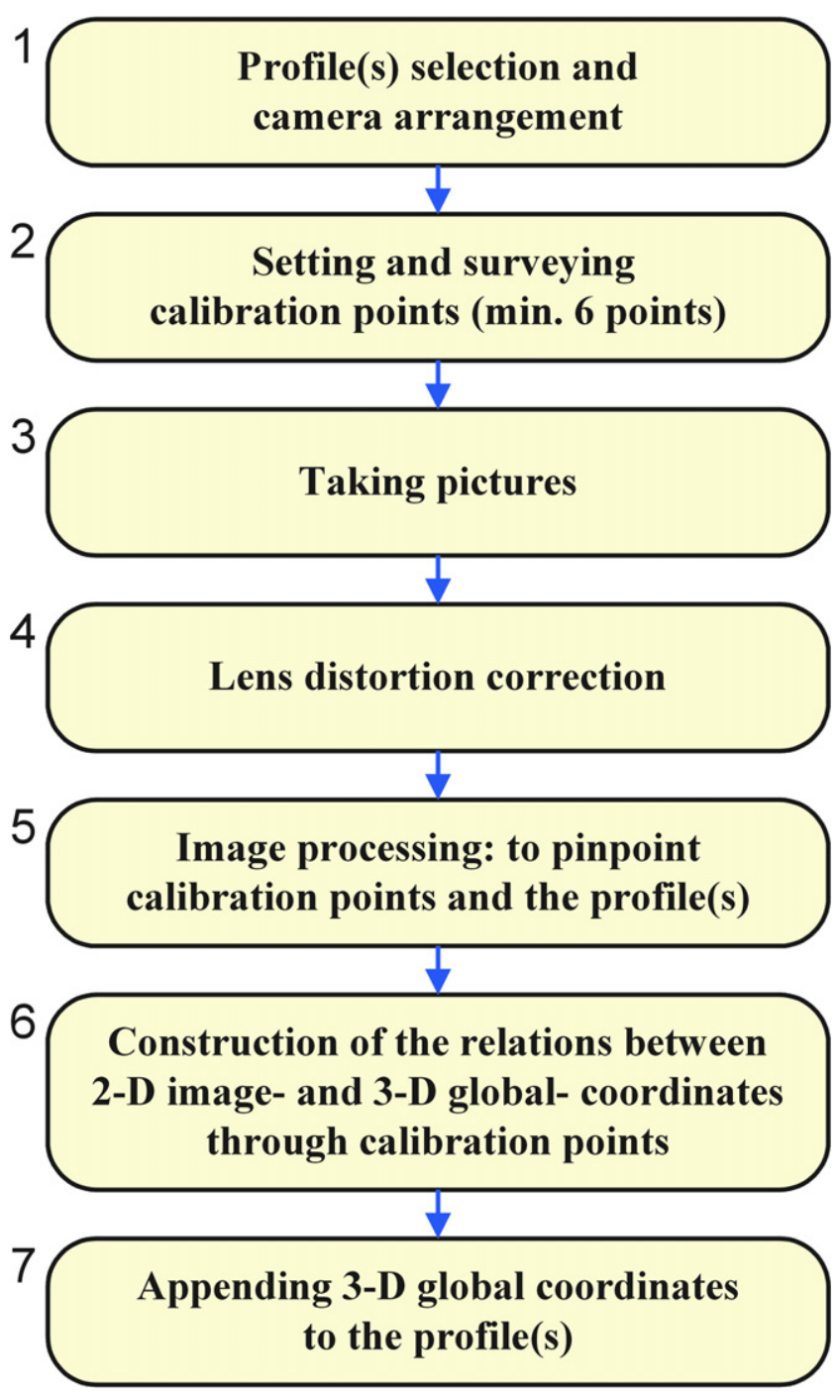

Fig. 3. The procedures for applying profile-image method to the survey of tunnel wall.

the taken image, a special technique is thus used, as in this study. Multi-images using the same calibration points, sometimes up to 200 images or more, were taken for the same profile and are then superimposed so that the contrast of the profile can be significantly improved, and points along the profile can be reliably extracted by the later image processing procedures as a result.

Keeping the above-mentioned concerns in mind, the validations are set to have two stages, as shown in Fig. 4: (1) Stage I - smallscale tunnel (Fig. 5); and (2) Stage II - medium size tunnel (Fig. 6). The small and the medium model tunnels are $1.0 \times 0.7 \times 1.0 \mathrm{~m}$ (length $\times$ width $\times$ height) and $5.0 \times 1.9 \times 3.3 \mathrm{~m}$, and are made of stainless thin-sheet and wood, respectively. The small tunnel, denoted as Model Tunnel I herein, is chosen for two reasons: (1) all the coordinates within it can be more precisely measured; and (2) the tunnel wall is flexible so that deformation with pre-specified magnitude can be applied. Therefore, the profile-image method can be further tested by checking the profiles before and after deformation. The medium size tunnel, denoted as Model Tunnel II, is chosen since its size, although being quite small indeed, is closer to that of the actual tunnel. Accordingly, for medium model tunnel, stronger laser should be employed and only geodetic device can be used for determining the coordinate of calibration points and profiles. The effect of image superimposed method is to be validated as well.
Concerning the later application for tunnel inspection, the equipments adopted should be simple and commonly available. Therefore, an ordinary digital camera, Canon EOS 350D with EF series $18-55 \mathrm{~mm}$ lens, is adopted for taking the photos. Both point laser and line laser were used to beam profiles inside a tunnel. These laser devices have a wavelength of $650 \mu \mathrm{m}$, respectively, with 12 and $5 \mathrm{~mW}$ powers for line laser and point laser. To determine the global 3D coordinates of the calibration points, a geodetic device of total station type is utilized. The adopted total station, Leica TCR 705, has a nominal angle precision of 5", 1.5 mgon and distance precision of $3 \mathrm{~mm}+2 \mathrm{ppm}$ in $170 \mathrm{~m}$ without target reflector. And for comparison purposes, a 3D terrestrial laser scanner RIEGL LMS-Z360i is also paralleley used to obtain tunnel profiles. Later on, the profiles obtained by the proposed profile-image method and terrestrial laser scanner will be compared to highlight the characteristics of these two methods.

The actual profiles of Model Tunnels I and II are, respectively, measured by a ruler and a total station. Several repetitions of measurements have been carried out to ensure the accuracy of the actual profiles of these two tunnels, which will serve as the basis for justifying the precision of other measuring methods. The coordinates of calibration points, highlighted by the brighter spots shown in Fig. 5b and by the numbered crosses shown in Fig. 6, are determined by the same ways. There are 105 calibration points arranged for Model Tunnel I and the other 36 points for Model Tunnel II. To enhance the precision of profile-image method, more than six calibration points, usually up to $10-15$ points nearer to the profile are used for determining the profile to be measured.

\section{Results of validation}

There are 10 profiles scanned by line laser and each of them is pictured by one single image, which will be further processed to determine their coordinates of each profile. A typical result, comparing to the actual profile, is depicted in Fig. 7a. These two profiles are so close to one another and are difficult to distinguish the discrepancies between them. Accordingly, a measure of mean discrepancy based on root mean square errors (RMSE) method is adopted to represent these discrepancies:

$R=\frac{1}{n} \sum_{i=1}^{n} R_{i}^{2}=\frac{1}{n} \sum_{i=1}^{n}\left(R_{\mathrm{m}}-R_{\mathrm{a}}\right)^{2}$

where $R_{\mathrm{m}}$ and $R_{\mathrm{a}}$ are the measured and the actual distance to a preset reference point, as shown in Fig. $7 \mathrm{~b} ; R_{\mathrm{i}}$ is then the error of an individual point along the profile to be measured, and is denoted as a function of an angle $\theta$ measured from left spring line clockwise; and $R$ represents the overall, mean error for the entire profile constituted by the deduced $n$ points. Fig. 7b illustrates the definition and configuration of $R_{\mathrm{m}}, R_{\mathrm{a}}, R_{\mathrm{i}}$ and pre-set reference point corresponding to an actual profile.

\subsection{Model Tunnel I}

A typical variation of $R_{\mathrm{i}}$ along a measured profile is shown in Fig. 8. The $R_{\mathrm{i}}$ 's are less than $2 \mathrm{~mm}$ for the particular profile shown in Fig. 8. It has been observed that the error near the middle part of the crown $\left(\theta=50-130^{\circ}\right)$ is less than the ones outside this range, as shown in Fig. 8. The majority of the calibration points are arranged in the middle part of the crown of the Model Tunnel $I$ $\left(\theta=50-130^{\circ}\right)$ accounting for the less error. The calibration points also have closer spacing near the middle crown, yet being far spaced around the side walls $\left(\theta=14-50^{\circ}\right.$, and $\left.130-166^{\circ}\right)$, as shown in Figs. $5 \mathrm{~b}$ and $7 \mathrm{a}$. As shown in Table 1 , a smaller profile $(\theta=45-$ $135^{\circ}$ ), which is deduced based on closer spaced calibration points, tends to have a better precision than the one $\left(\theta=14-166^{\circ}\right)$ with 


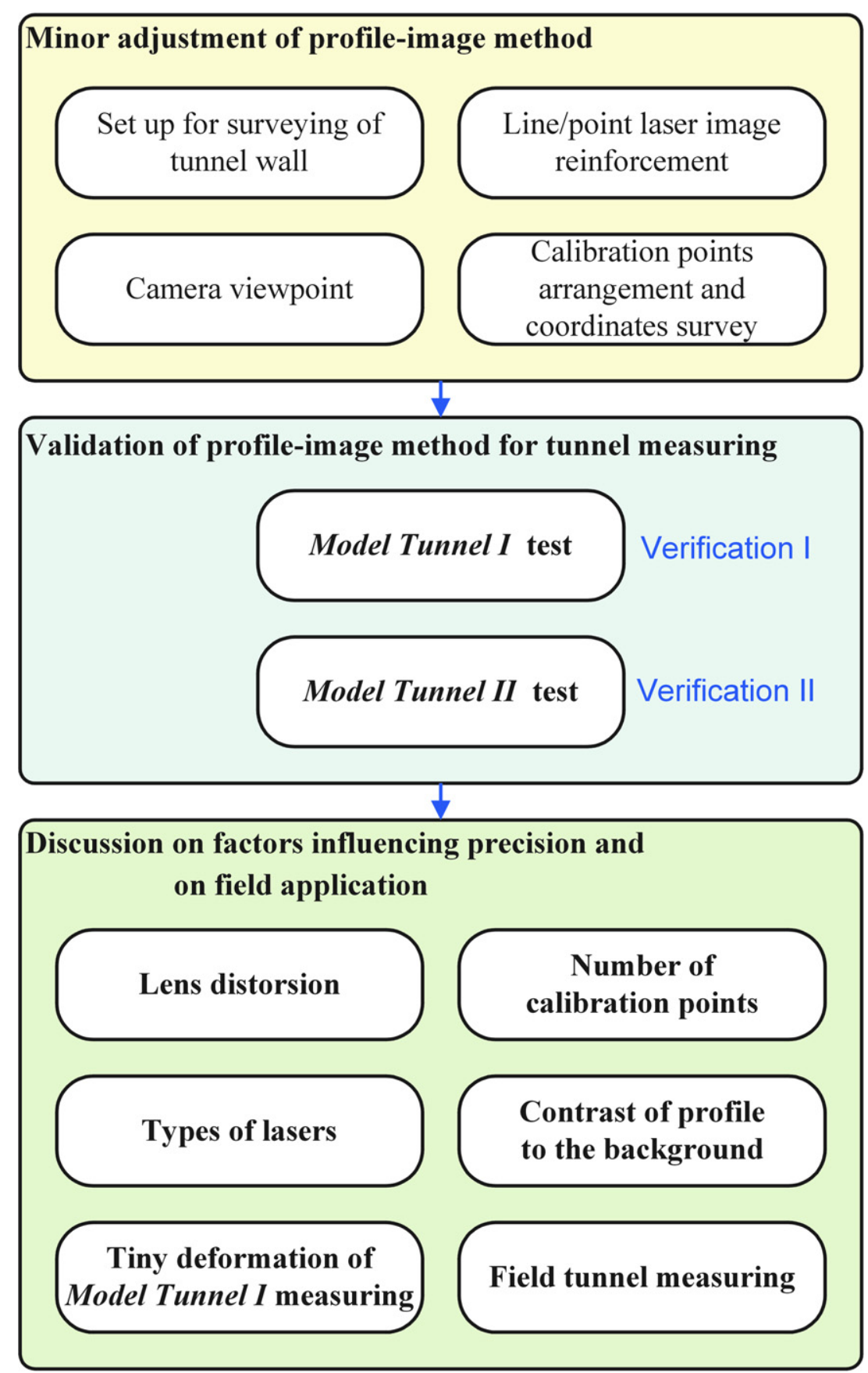

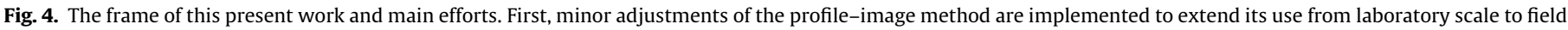

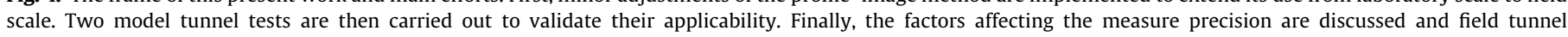
measurements are addressed.

far-spaced calibration points included in deduction process, as shown in the fourth and the third columns of Table 1, respectively. As a result, the overall $R$ for each profile conspicuously decreases to below $1.25 \mathrm{~mm}$ in most cases, if close-spaced calibration points are utilized.

Based on the $R_{\mathrm{i}}$ of each measured profile, the mean error of this profile, $R$, can then be calculated using Eq. (4), and the $R$ 's for the 10 measured profiles are summarized in Table 1 . The profiles are numbered to according their distance to the tunnel end nearer the camera; a close profile has a smaller number, that is, Profile \#1 is closest to the camera. Being closest to the camera, the upper profile is thus located at the upper end of the image, where greater lens distortion can be expected. This phenomenon may account for the greatest $R(=1.69 \mathrm{~mm})$ among the 10 profiles. Meanwhile, the calibration points are not located right on Profile \#1, which may further worsen the results of deduction.

For a same profile, more than one photo were taken and compared. Ideally, these images are supposed to be exactly the same and so is the deduced profile. These images are further superimposed and the deduced profile is then compared to the actual profile, as shown in Fig. 9. Accordingly, all the R's for the measured profiles (based on superimposed images) are calculated and summarized in the fifth column of Table 1 . The R's deduced from superimposed line laser images, with an average of $1.20 \mathrm{~mm}$ and a 


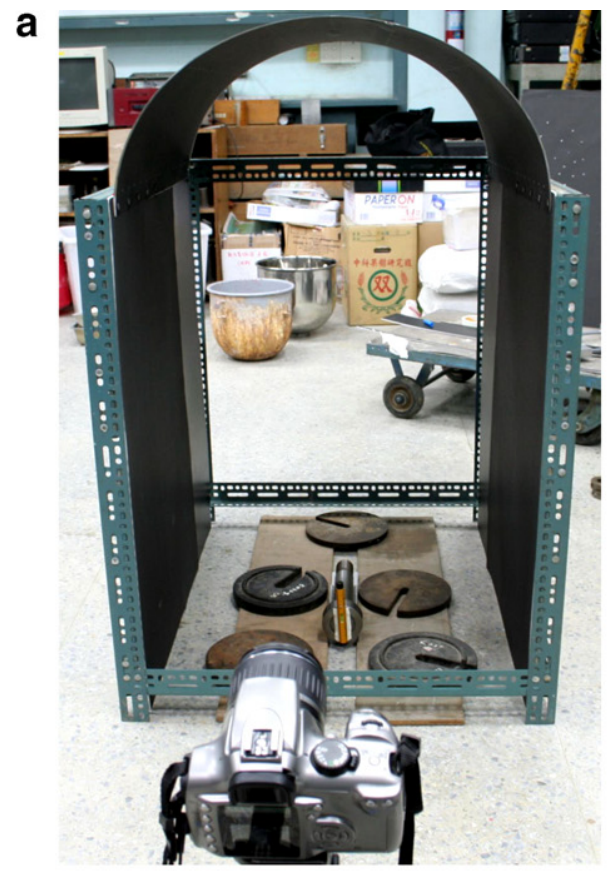

b

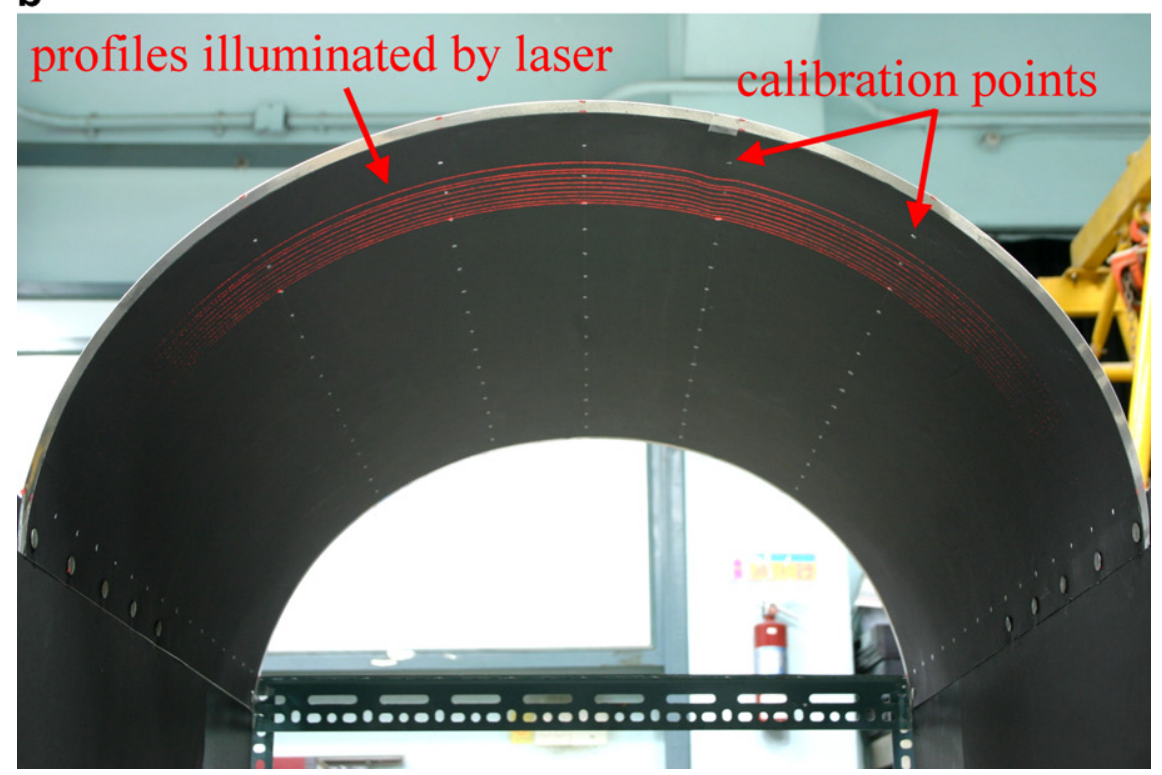

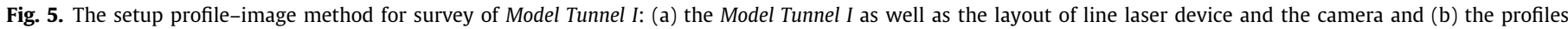
illuminated by laser and the arranged 105 calibration points.

standard deviation of $0.68 \mathrm{~mm}$, are both similar to those deduced from single line laser image, which have an average of $1.15 \mathrm{~mm}$ and a standard deviation of $0.22 \mathrm{~mm}$. As such, superimposing of images is acceptable and will be used for a larger tunnel to emphasize the contrast of the laser illumination.

For a larger tunnel, the illumination of line laser is not sufficient to illuminate a detectable profile; therefore, a point laser must be used. By rotating the point laser, taking photos for the points along a profile and superimposing the images, an image containing all the points can be obtained and the profile can then be deduced. The number of images to be superimposed can be up to 250 for each profile. The precision of point laser should be tested using Model Tunnel I before applying it to a larger tunnel. The typical variation of $R_{\mathrm{i}}$ along a profile is shown in Fig. 10, which has a maxi- mum $R_{\mathrm{i}}$ of $2.72 \mathrm{~mm}$. Furthermore, all the $R$ 's for the six measured profiles are calculated and summarized in Table 2 . In general, by comparing Table 2 with Table 1, the profiles deduced from point laser have somewhat greater, yet acceptable error.

The variation of $R$ for distinct profiles obtained by previously mentioned three methods (single image and superimposed image using line laser and point laser) is compared and illustrated in Fig. 11. For these profiles of Model Tunnel I, the measured results are rather stable and seem to be less than $2 \mathrm{~mm}$ in most cases, which is acceptable for safety inspection of tunnel, although better precision is still wished. Overall, the results deduced from single image are better than those from superimposed images, and the results obtained by line laser are better than those obtained by point laser. 


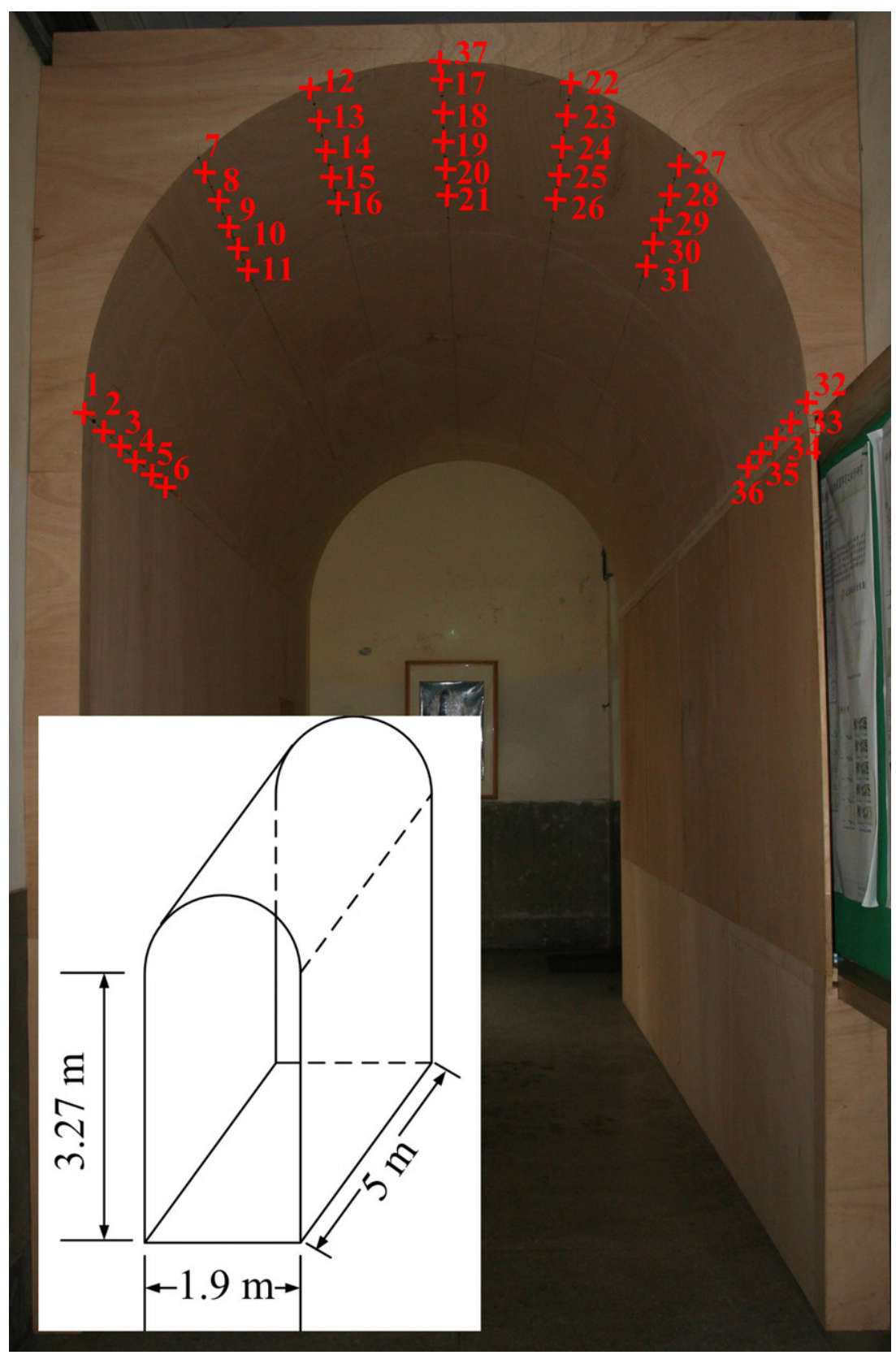

Fig. 6. The Model Tunnel II. The 36 calibration points are highlighted by numbered crosses.

\subsection{Model Tunnel II}

Since Model Tunnel II is larger than Model Tunnel I, the illumination of line laser is not sufficient to provide a clear image for deducing the profile. A trial was made to rotate a point laser to yield an illuminated profile, and it was found that the illumination is still not sufficient. Finally, superimpose of images, whether obtained by line laser or point laser, must be used for the Model Tunnel II. Therefore, the coordinates of profiles deduced from superimposed images by line laser and by point laser are discussed as follows.

When utilizing the superimposed image technique, there are 12 profiles illuminated by line laser and another 10 profiles by point laser conducted. Among which two typical results, compared to their correspondingly actual profiles, are illustrated in Figs. 12a and 13a. While taking a photo, a minor vibration may be caused while shutting the picture. Unfortunately, line laser is very sensitive to such shutting vibration such that interlacing of illuminated profiles can be found within a superimposed image. Therefore, some pixels, outside the range of profile, are removed to avoid mistake when conducting point identification. As a result, the distribution of points determined from line laser (Fig. 12a) is less uniform than the cases obtained by point laser (Fig. 13a).

The typical variations of $R_{\mathrm{i}}$ along a measured profile, using line laser and point laser, are, respectively, shown in Figs. 12b and 13b. All the R's for these measured profiles are then calculated and summarized in Table 3. The profiles illuminated by point laser, with $R$ 's ranging 2.50-4.16 $\mathrm{mm}$ and an average of $3.25 \mathrm{~mm}$, are better than those obtained by line laser, having the $R$ 's ranging $3.35-7.33 \mathrm{~mm}$ and an average of $4.86 \mathrm{~mm}$. The relative strong illumination of point laser may account for the better precision.

The measured results for Model Tunnel II show that the R's of profile-image method are $3-5 \mathrm{~mm}$ in most cases, as shown in Table 3. It is seen that a larger tunnel tends to have larger $R$ 's, comparing Table 3 (Model Tunnel II) to Tables 1 and 2 (Model Tunnel 


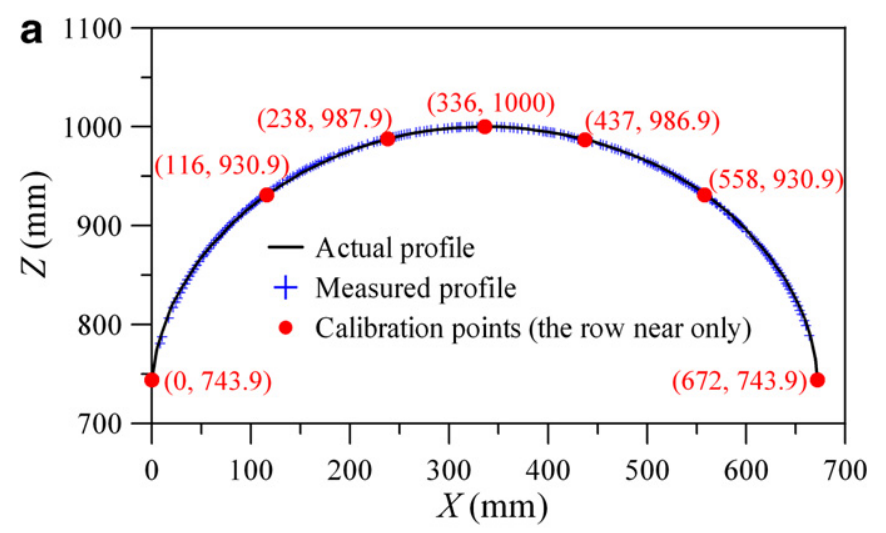

b

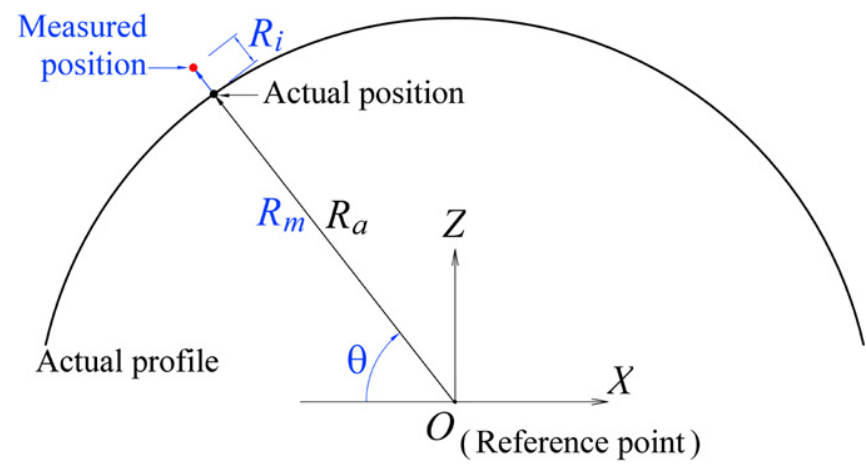

Fig. 7. A typical result of Model Tunnel I deduced from profile-image method, profile illuminated by line laser and pictured by one single image, is compared to the actual profile (Fig. 7a). These two profiles are too close to distinguish the discrepancies in between. Therefore, additional definition and configuration of $R_{\mathrm{m}}$, $R_{\mathrm{a}}, R_{\mathrm{i}}$ and pre-set reference point corresponding to an actual profile are used for comparison propose (Fig. $7 \mathrm{~b}$ ).

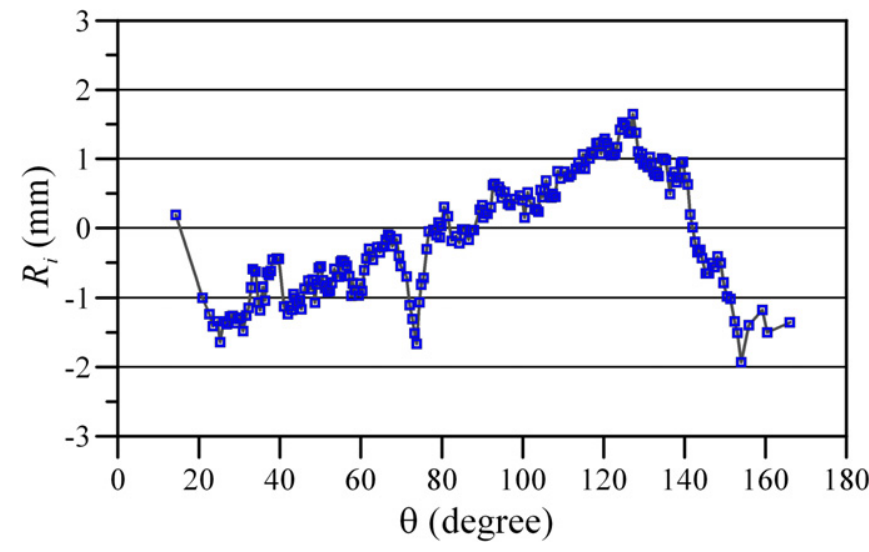

Fig. 8. A typical variation of $R_{\mathrm{i}}$ along a measured profile, deduced from single line laser image, of Model Tunnel $I$.

I). These results validate the profile-image method for measuring tunnel wall. If the deformation of tunnel has the order of cm's, then the precision of $3-5 \mathrm{~mm}$ seems to be acceptable.

For comparison purposes, the profile obtained by laser scanner is also conducted, as shown in Fig. 14a. Since the points scanned by laser scanner are not necessarily located on one profile, the profile is obtained by treating the points, located within $10 \mathrm{~mm}$ distance to the measured profile, to be on the same profile. Fig. 14b displays the corresponding variation of $R_{\mathrm{i}}$ along the profile. The $R$ 's of the profile determined from laser scanner, ranging $10.02-13.68 \mathrm{~mm}$
Table 1

Root mean square error for survey of Model Tunnel I, profiles illuminated by line laser

\begin{tabular}{lrlll}
\hline Profile no. & $Y^{\mathrm{a}}(\mathrm{mm})$ & $R^{\mathrm{b}}(\mathrm{mm})$ & \\
\cline { 3 - 5 } & & $\theta=14-166^{\circ}$ & $\theta=45-135^{\circ}$ & \\
\cline { 3 - 5 } & & Single image & Single image & Superimposed image \\
\hline 1 & 5.9 & 1.94 & 1.69 & 2.76 \\
2 & 30.4 & 1.82 & 1.01 & 1.42 \\
3 & 50.9 & 1.52 & 1.24 & 1.66 \\
4 & 70.5 & 1.79 & 1.04 & 0.63 \\
5 & 90.2 & 1.39 & 1.08 & 1.01 \\
6 & 110.2 & 1.54 & 0.98 & 1.02 \\
7 & 130.5 & 1.56 & 1.20 & 0.86 \\
8 & 148.9 & 1.63 & 1.25 & 0.83 \\
9 & 171.1 & 1.25 & 1.08 & 0.64 \\
10 & 204.3 & 1.67 & 0.90 & - \\
Average & & 1.61 & 1.15 & 1.20 \\
Standard dev. & & 0.21 & 0.22 & 0.68 \\
\hline
\end{tabular}

${ }^{\text {a }} \mathrm{Y}$ is the distance of the profile to the portal. The camera is located outside the tunnel.

${ }^{\mathrm{b}} R$ is the mean error, represented by the root mean square error (RMSE) as defined by Eq. (4), of the measured distance from the actual distance for all points along a profile.

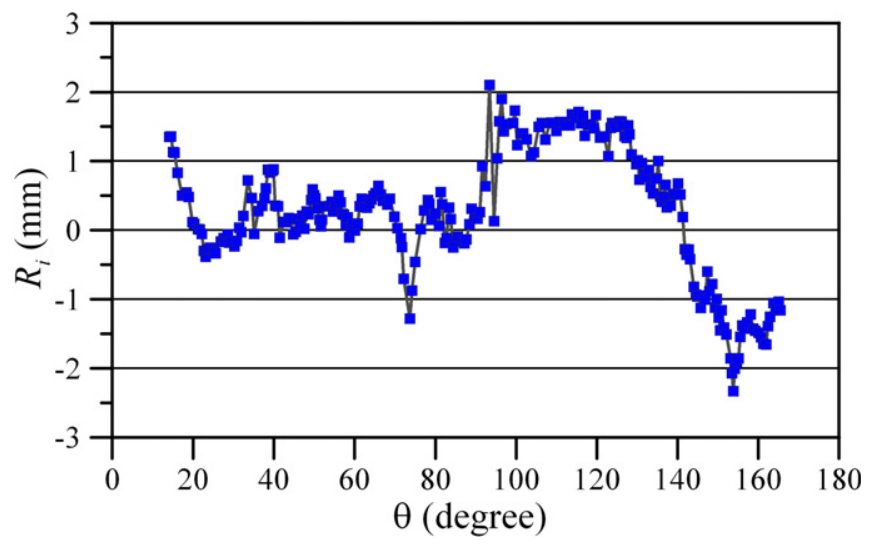

Fig. 9. A typical variation of $R_{\mathrm{i}}$ along a measured profile, deduced from superimposed line laser image, of Model Tunnel $I$.

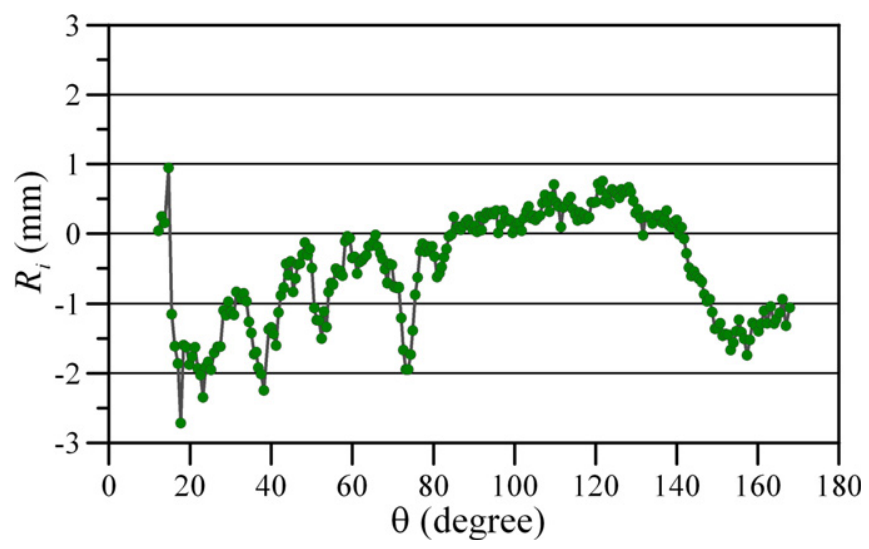

Fig. 10. A typical variation of $R_{\mathrm{i}}$ along a measured profile, deduced from superimposed point laser image, of Model Tunnel $I$.

and with an average of $11.79 \mathrm{~mm}$, are greater than those obtained from the proposed profile-image method, as summarized in Table 4. This $10-13 \mathrm{~mm}$ error is in fact the precision of the adopted laser 
Table 2

Root mean square error for survey on middle crown $\left(\theta=45-135^{\circ}\right)$ of Model Tunnel I, profiles illuminated by rotating point laser and composed of superimposed images

\begin{tabular}{lcc}
\hline Profile no. & $Y(\mathrm{~mm})$ & $R(\mathrm{~mm})$ \\
\hline 1 & 15.6 & 2.22 \\
2 & 28.6 & 1.63 \\
3 & 44.4 & 1.64 \\
4 & 79.3 & 1.57 \\
5 & 98.2 & 1.31 \\
6 & 116.8 & 0.79 \\
Average & & 1.53 \\
Standard dev. & & 0.47 \\
\hline
\end{tabular}

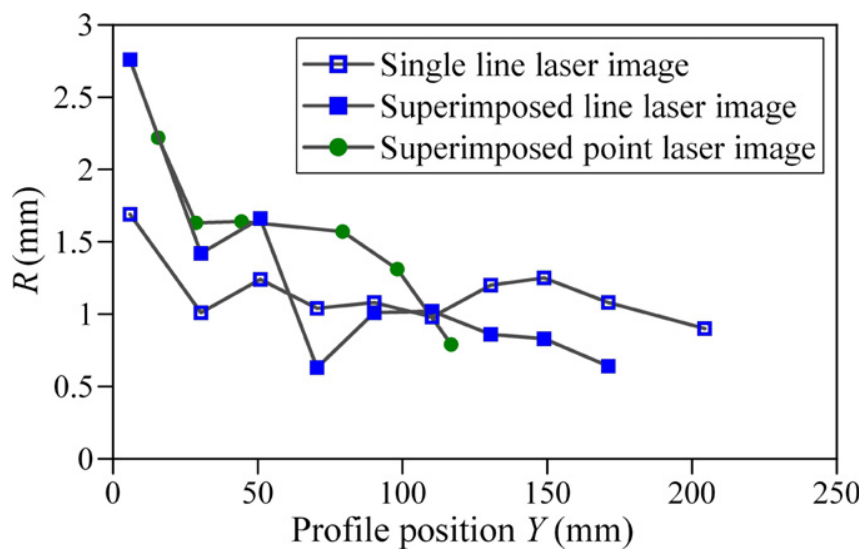

Fig. 11. The variation of $R$ for distinct profiles, deduced from single images and superimposed images using line laser and point laser, of Model Tunnel $I$. The results obtained by profile-image method are rather stable and seem to be less than $2 \mathrm{~mm}$ in most cases

scanner. Therefore, a better precision can be made if a more precise laser scanner is used.

\section{Discussion}

As perceived from the previous tests, the factors influencing the precision of the proposed profile-image method may at least include distortion of lens, point identification, numbers of calibration points, types of lasers as well as contrast of profile to the background.

Lens distortion, often possessed by ordinary digital camera, makes a point to be deviated from the correct point in an image and leads to incorrect deduced coordinates. In this study, the influence of lens distortion has been minimized by adjusting the taken image to a less distorted image. The adjustment was based on the distortion nature of the lens used, and was conducted using the software provided by Caltech (Savarese et al., 2005). Without using this adjustment process, the error can increase from 12 to $26 \mathrm{~mm}$ for the profiles of Model Tunnel I.

Given a laser-illuminated point in an image, there can be a group of pixels in the image, and the selection of right pixel inherently affects the precision of the coordinates deduced.

To properly determine the location of a "point" within a group of pixels, the technique proposed by Veber et al. (1997) is adopted: The obscured laser spots on the image can be refined to sub-pixel by fitting a second-degree interpolation surface around the spot. As a result, the expected RMSE on the $\left(x^{s}, y^{s}\right)$ image coordinates obtained by this technique has the order of 0.25 pixel. The laser spots in an image usually have the size of 4-7 pixels. Each pixel represents a distance of $0.5-0.75 \mathrm{~mm}$ in tunnel according to the resolution of image taken. Based on the adopted image processing
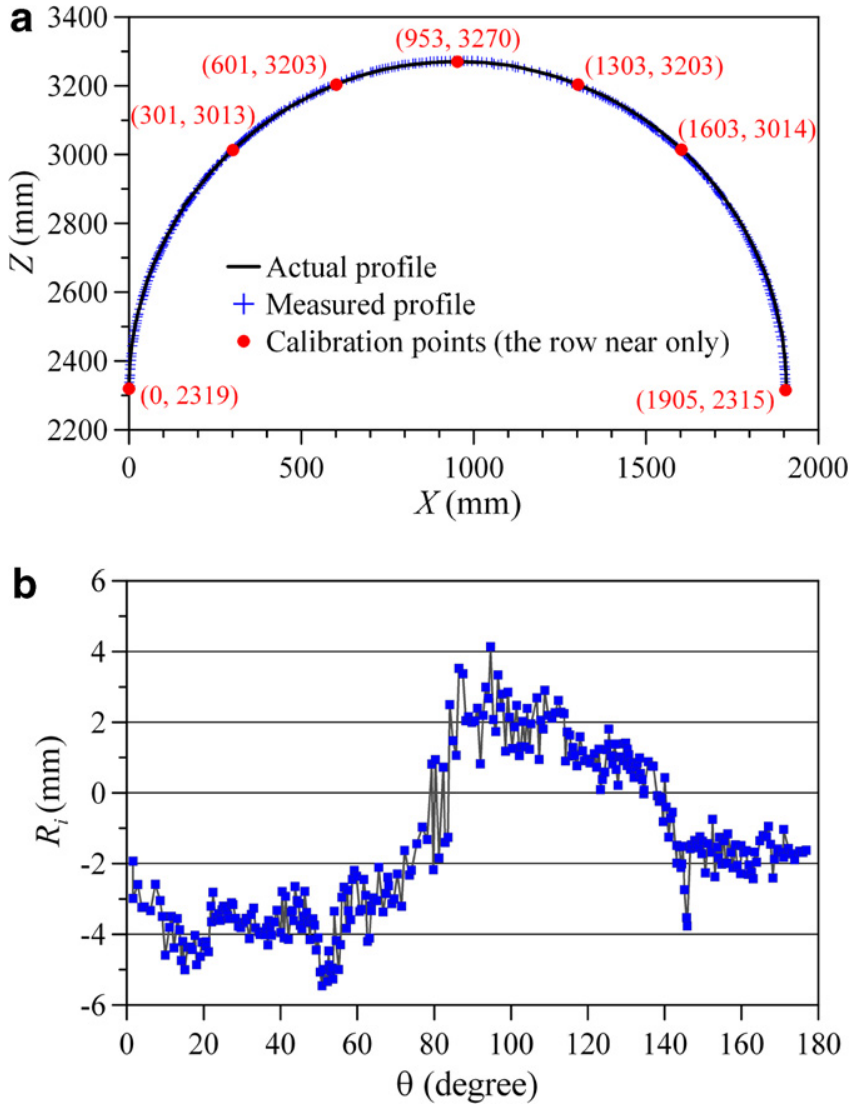

Fig. 12. A typical result of Model Tunnel II deduced from superimposed line laser image: (a) the measured profile and the correspondingly actual profile and (b) the variation of $R_{\mathrm{i}}$ along the profile.

technology, the accuracy for pinpointing is about of the order of 0.25 pixel. Therefore, the theoretical accuracy in determining the coordinates, induced by locating the pixel alone, is about the order of $0.25-0.56 \mathrm{~mm}$. The measured R's for Model Tunnel I ( 1.53 mm), with other sources of error involved, are about 2-6 times of the theoretical accuracy.

The rest of the three issues affecting the precision of imaging method are discussed as follows.

\subsection{Number of calibration points}

As previously mentioned, at least six calibration points are required to determine the profile. It is of interest to know (1) Will the precision be improved by increasing the number of calibration points? (2) How many calibration points are needed to yield a reliable profile?

To evaluate the influence of the number of calibration points on the precision, three points within a profile are selected for further comparison. These three points are located at the positions of $\theta=45^{\circ}, 60^{\circ}$ and $90^{\circ}$, respectively. They are chosen because relatively greater $R_{\mathrm{i}}$ 's are observed, as shown in Fig. 13. In addition to the profile obtained using six calibration points, $7,10,14,21$, 28 and 36 calibration points around the measured points are used to determine their coordinates. As a result, the variations of $R_{\mathrm{i}}$ of the three points with the number of calibration points are obtained, as illustrated in Fig. 15. If the error on the plane of profile is concerned, namely $2 \mathrm{D}$ situation, the $R_{\mathrm{i}}$ reduces to a constant level when more than 7 points are used (Fig. 15a). When the error in $3 \mathrm{D}$ space is of concern, it takes $10-14$ points to reduce the error to a low, constant level, as shown in Fig. 15b. This test also highlights 

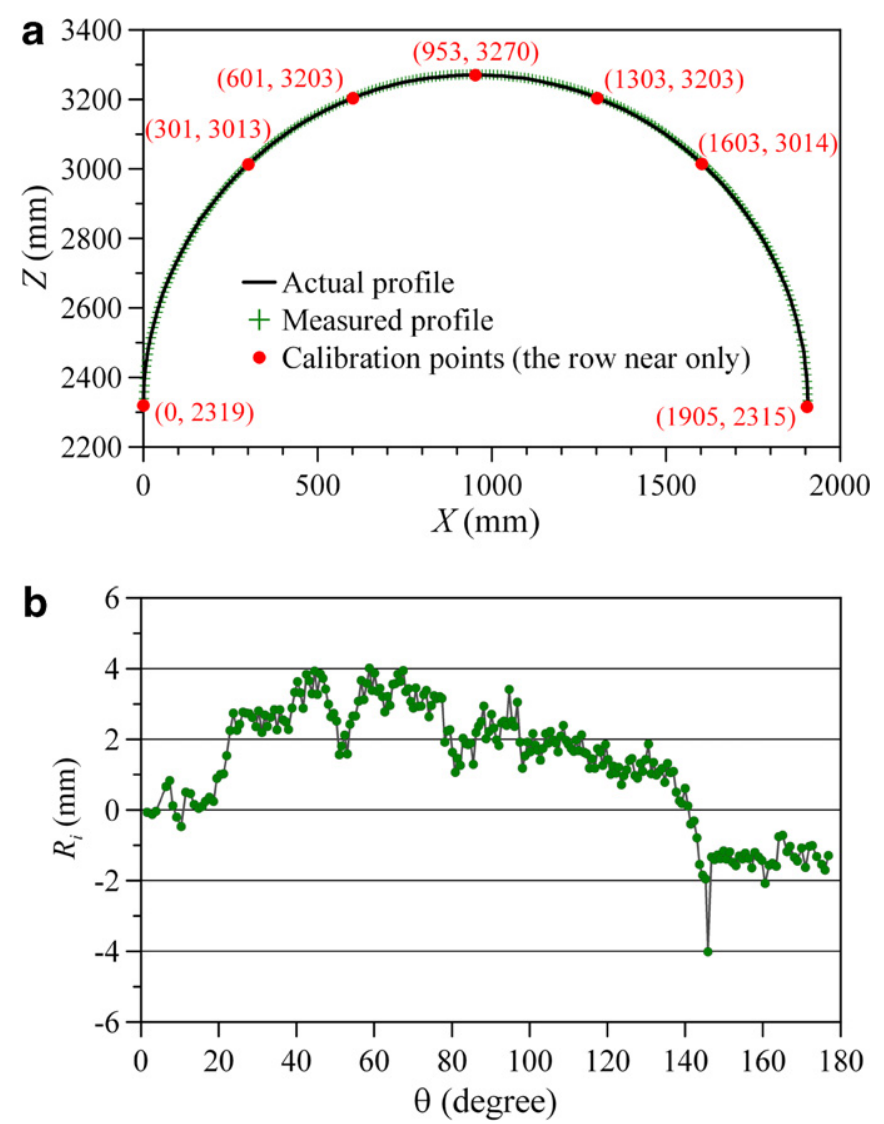

Fig. 13. A typical result of Model Tunnel II deduced from superimposed point laser image: (a) the measured profile and the correspondingly actual profile and (b) the variation of $R_{\mathrm{i}}$ along the profile.

Table 3

Root mean square error for survey of Model Tunnel II, profiles composed of superimposed images

\begin{tabular}{lcccccc}
\hline \multicolumn{2}{l}{ Profile illuminated by line laser } & & \multicolumn{3}{l}{ Profile illuminated by point laser } \\
\cline { 1 - 2 } \cline { 6 - 7 } Profile no. & $Y(\mathrm{~mm})$ & $R(\mathrm{~mm})$ & & Profile no. & $Y(\mathrm{~mm})$ & $R(\mathrm{~mm})$ \\
\hline 1 & 51.7 & 4.22 & & 1 & 45.3 & 3.29 \\
2 & 103.4 & 3.92 & & 2 & 75.7 & 2.98 \\
3 & 148.5 & 3.98 & & 3 & 103.3 & 2.83 \\
4 & 195.0 & 3.35 & & 4 & 159.9 & 2.50 \\
5 & 251.6 & 3.40 & & 5 & 232.2 & 2.73 \\
6 & 302.7 & 5.19 & & 6 & 282.0 & 3.44 \\
7 & 352.1 & 5.72 & & 7 & 333.5 & 3.97 \\
8 & 393.7 & 4.67 & & 8 & 394.0 & 2.74 \\
9 & 448.9 & 5.27 & & 9 & 465.8 & 3.88 \\
10 & 498.5 & 5.48 & & 10 & 495.6 & 4.16 \\
11 & 553.4 & 5.80 & & & \\
12 & 607.6 & 7.33 & & & \\
Average & & 4.86 & & & & \\
Standard dev. & & 1.16 & & & & \\
\hline
\end{tabular}

that (1) 3D situation requires more calibration points than 2D situation; (2) Too many calibration points do not help increasing the precision.

\subsection{Types of lasers}

Although line laser and point laser have about the same precision for Model tunnel I, point laser seems to have much better precision for a greater tunnel (Model Tunnel II), as shown in Fig. 16.

The interlaced phenomenon existing in superimposed line laser images obstructs proper image processing and evidently reduces
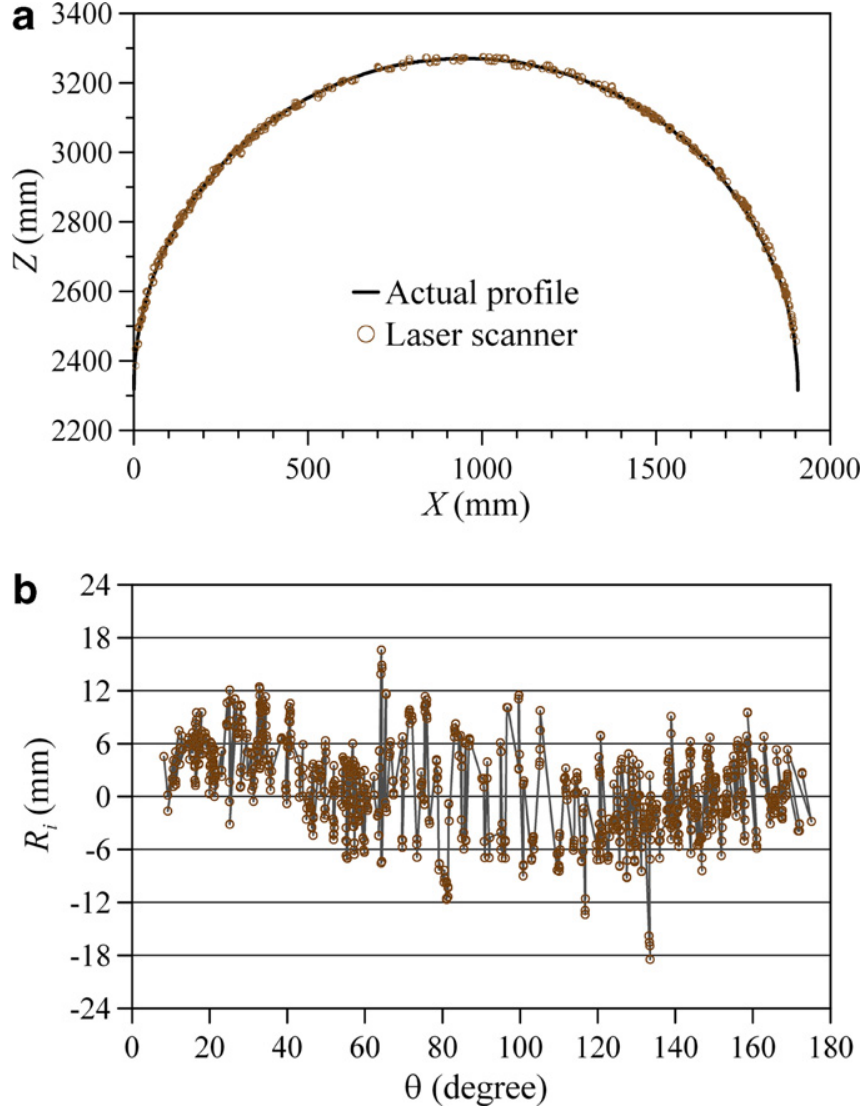

Fig. 14. A typical result of Model Tunnel II obtained from laser scanner: (a) the measured profile and the correspondingly actual profile and (b) the variation of $R_{i}$ along the profile.

Table 4

Root mean square error for survey of Model Tunnel II, deduced from results obtained by RIEGL LMS-Z360

\begin{tabular}{lcc}
\hline Profile no. & $Y(\mathrm{~mm})$ & $R(\mathrm{~mm})$ \\
\hline 1 & 45.3 & 10.10 \\
2 & 75.7 & 10.02 \\
3 & 103.3 & 10.14 \\
4 & 159.9 & 10.30 \\
5 & 232.2 & 10.80 \\
6 & 282.0 & 12.81 \\
7 & 333.5 & 13.68 \\
8 & 394.0 & 12.81 \\
9 & 465.8 & 12.72 \\
10 & 495.6 & 12.66 \\
11 & 553.4 & 12.67 \\
12 & 607.6 & 12.77 \\
Average & & 11.79 \\
Standard dev. & & 1.38 \\
\hline
\end{tabular}

its precision. Consequently, conjunction use of point laser and superimpose of images are found to be more feasible in practice.

\subsection{Contrast of profile to the background}

Laser-lit spots with relatively high contrast to the background, which can be acquired by prolonging the exposure time while taking images, are ideal for profile-image method to pinpoint their positions. Nevertheless, prolonging the exposure time may have some disadvantages, including (1) the dim corona around a spot can be made brighter so that the size of spot can be increased, 

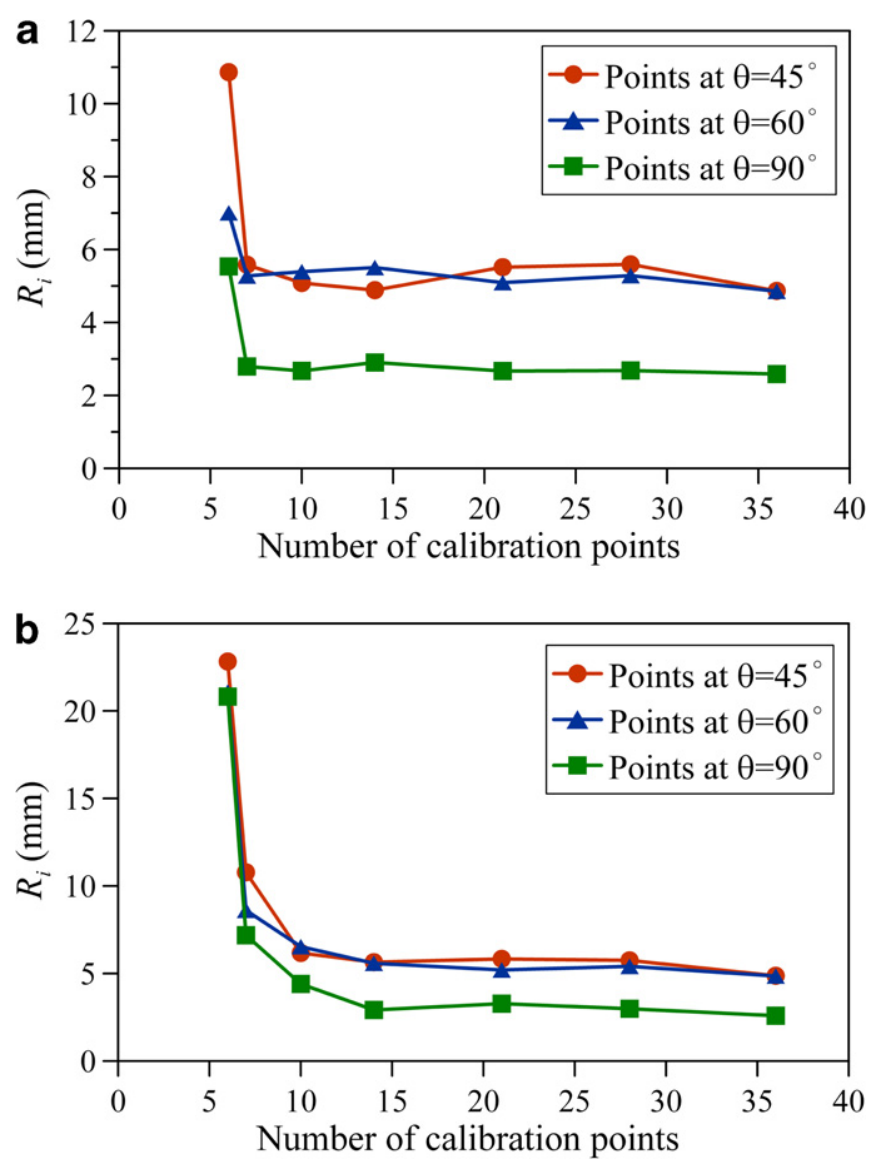

Fig. 15. The variations of $R_{\mathrm{i}}$ of three pre-set points with the number of calibration points which are used to deduce their coordinates: (a) the $R_{\mathrm{i}}$ reduces to a constant level when more than 7 points are used in case the error on the plane of profile (2D) is concerned and (b) the $R_{\mathrm{i}}$ reduces to a low, constant level as 10-15 points are used in case the error in 3D space is concerned.

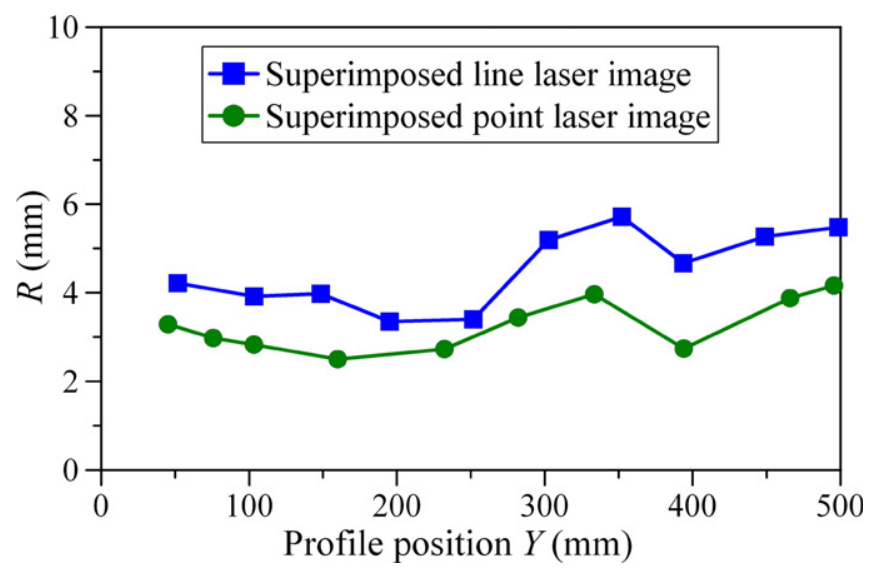

Fig. 16. The variation of $R$ for distinct profiles, deduced from superimposed images using line laser and point laser, of Model Tunnel II. Contrasting to Fig. 11, profiles illuminated by point laser seem to have much better precision for a greater tunnel.

and (2) the undesirable spots, caused by scattering reflection of laser or background light sources, are also enhanced simultaneously. These unfavorable effects may lead to a lower precision, or, even worse, mistaking of points. Therefore, the factors affecting the contrast to the background, such as the exposure time and background illumination, are further studied to reveal their influence on the precision of profile.

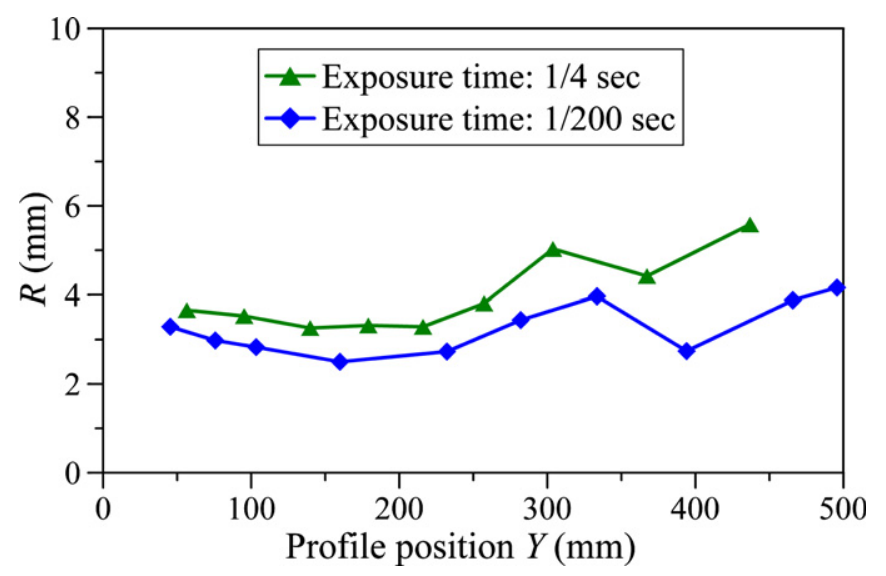

Fig. 17. The influence of exposure time. The R's for various profiles obtained from exposure time of $1 / 200 \mathrm{~s}$ are consistently less than those of $1 / 4$.

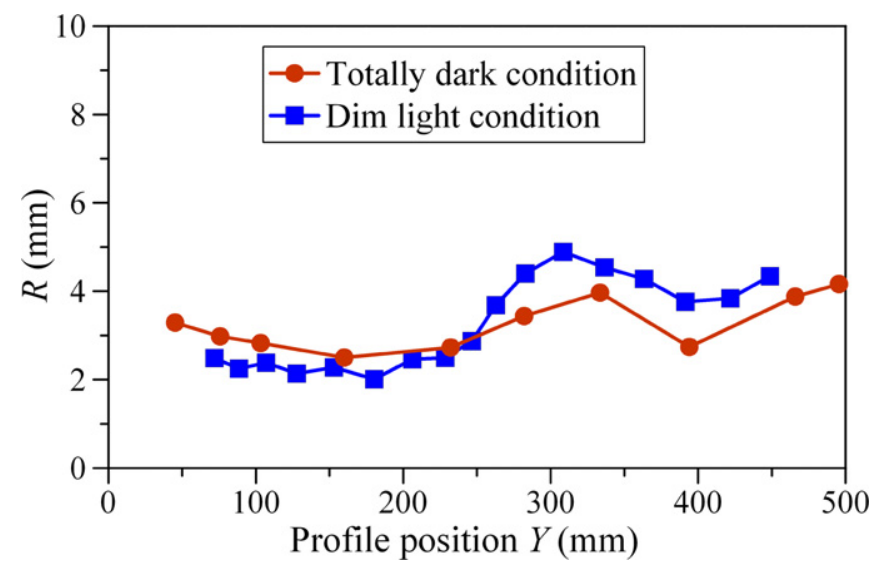

Fig. 18. The influence of background illumination. Since the effect of background illumination is compensated by the exposure time, the results obtained for totally dark condition are about the same order of precision to those for dim light condition.

The influence of exposure time is illustrated in Fig. 17. Since smaller laser spots with sufficient contrasts are provided, the $R$ 's for various profiles of Model Tunnel II (superimposed images using point laser) obtained from the exposure time of $1 / 200 \mathrm{~s}$ are consistently less than those of $1 / 4$. The most appropriate exposure time is not necessarily the same for different tunnels; therefore, the exposure time should be properly determined before conducting safety inspection so that the best precision can be achieved.

The R's obtained from totally dark condition in nighttime and from dim lit condition at nightfall are compared in Fig. 18. Supposedly, since there is no background illumination in the totally dark condition and no interference would happen, better precision should be obtained for totally dark condition. However, about the same orders of precision were obtained for both cases. For the totally dark condition, longer exposure time is required owing to the totally dark background, the laser-lit spot is broadened, and the precision is accordingly reduced, that is, the effect of better background condition (totally dark) is compensated by the longer coexposure time.

\subsection{Application to the survey of tunnel wall}

Given the precision of about $1.2 \mathrm{~mm}$ for Model Tunnel I, it is of interest to know whether the deformation and the corresponding precision of tunnel wall can be detected. For this purpose, an 


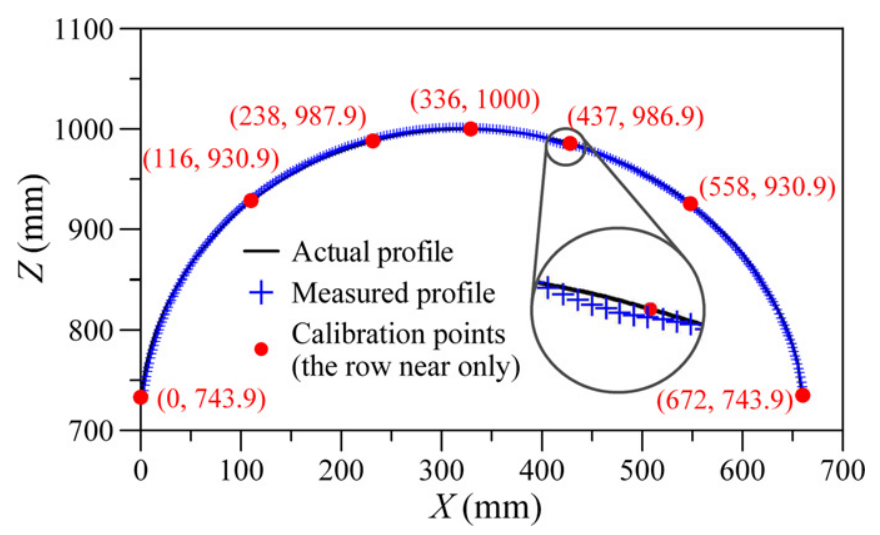

Fig. 19. The application of local tiny deformation detection. The proposed profileimage method has effectively showed the deformation of tunnel wall, even the deformation is rather small.

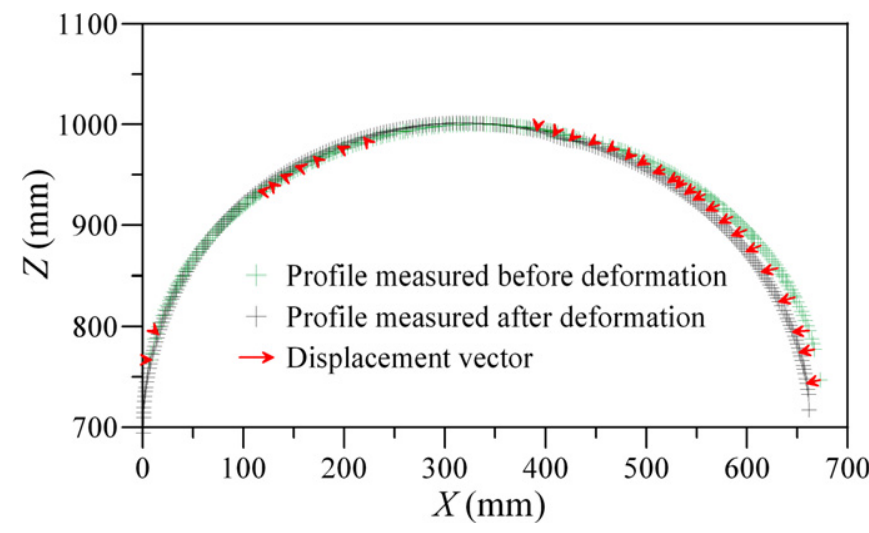

Fig. 20. The application of deformation detection. The deformation pattern has been successful revealed by the two successfully measured profiles.

intentional bulge with thickness of only approximately $3 \mathrm{~mm}$ is designed to provide a slight deformation of tunnel wall. The resulted profile and the original profile are compared, as shown in Fig. 19. It can be seen that the proposed imaging method has effectively showed the deformation of tunnel wall, even the deformation is rather small.

Furthermore, the Model Tunnel I is forced to deform with maximum horizontal displacement of about $15 \mathrm{~mm}$ on its right side. One of the measured profiles is compared with its original profile, as illustrated in Fig. 20. The deformation pattern has been successfully revealed by the two profiles measured. Such information can be very helpful for identifying deformation mechanism later. Moreover, the data points on the deformed segment are about 250 , which are much more than those provided by geodetic survey. Therefore, the profile-image method shows merits, both in the precision and in the numbers of points along a profile, in characterizing the whole deformed pattern of the profile, when compared to methods using conventional geodetic survey or laser scanner.

The proposed profile-image method has also been tested in two real, full size tunnels. For the first tunnel, a double-lane highway tunnel, it took about half day to survey six profiles with all procedures included. For the second tunnel, a single-lane railway tunnel, it took about $2.5 \mathrm{~h}$ to measure three profiles. Most of the time was used for surveying the coordinates of calibration points. In fact, the time needed for measuring a particular profile is just about 15$20 \mathrm{~min}$.

\section{Conclusive remarks}

Profile-image method has been developed for measuring profiles of moving objects in small, laboratorial scale. This study extends this method and tests its applicability for measuring the profile of a tunnel. The superimposed image technique is conjunctively utilized to enhance the insufficient contrast of the laser-lit profile as the tunnel size became larger. And results from experiments with small and medium scales prove that the proposed profile-image method exhibits merits in providing good precision, sufficient data points along a profile and fastness in time survey. Consequently, especially for tunnels in operation, the profile-image method can serve as a feasible and efficient way for safety inspection and for continuously monitoring the tunnel deformation. It is found that the precision of the proposed profile-image method can be influenced by the lens distortion, image resolution, types of lasers and contrast of profile to the background. This also highlights the key issues to be improved in the future.

\section{Acknowledgements}

The authors acknowledge the contribution of Mr. Chun-Wei Chao and Ms. Ya-Chu Chiu in completion of this work. The authors also thank two anonymous reviewers for their valuable suggestions to make this paper in the present form.

\section{References}

Boehler, W., Heins, G., Marbs, A., 2001. The potential of non-contact close range laser scanners for cultural heritage recording. In: Proceedings of CIPA International Symposium, Potsdam, Germany.

Boehler, W., Marbs, A., 2002. 3D scanning instrument. In: CIPA, Heritage Documentation - International Workshop on Scanning for Cultural Heritage Recording-Corfu, Greece.

Capart, H., Young, D.L., Zech, Y., 2002. Voronoï imaging methods for the measurement of granular flows. Experiments in Fluids 32 (1), 121-135.

Chao,C.W., 2006. Application of 3D-laser scanning technique in tunnel engineering. M.S. thesis, Grad. Inst. of Civil Eng., National Taiwan University, Taipei (in Chinese).

Chiu, Y.C., 2007. Tunnel profile measuring technology and traceback analysis of the incremental stress. M.S. thesis, Grad. Inst. of Civil Eng., National Taiwan University, Taipei (in Chinese).

Douxchamps, D., Devriendt, D., Capart, H., Craeye, C., Macq, B., Zech, Y., 2005. Stereoscopic and velocimetric reconstructions of the free surface topography of antidune flows. Experiments in Fluids 39 (3), 533-551.

Faugeras, O., Luong, Q.T., 2001. The Geometry of Multiple Images. MIT, Cambridge.

Jähne, B., 1995. Digital Image Processing. Springer, New York.

Japanese Society of Civil Engineers, 2002. The present state of construction and maintenance of mountain tunnel lining, Tokyo (in Japanese).

Japanese Society of Civil Engineers, 2003. Tunnel deformation mechanism, Tokyo (in Japanese)

Langer, D., Markus, M., Franz, H., Christoph, F., 2000. Imaging ladar for 3-D surveying and CAD modeling of real-world environments. International Journal of Robotics Research 19 (11), 1075-1088.

Lichti, D., Stewart, M.P., Tsakiri, M., Snow, A.J., 2000. Benchmark testing on a threedimensional laser scanning system. Geomatics Research Australasia 72, 1-23.

Ni,W.J., 2005. Groundwater drainage and recharge by geomorphically active gullies. M.S. thesis, Grad. Inst. of Civil Eng., National Taiwan University, Taipei.

Savarese, S., Chen, M., Perona, P., 2005. Local shape from mirror reflections. International Journal of Computer Vision 64 (1), 31-67.

Spinewine, B., Capart, H., Larcher, M., Zech, Y., 2003. Three-dimensional Voronoi imaging methods for the measurement of near-wall particulate flows. Experiments in Fluids 34 (2), 227-241.

Veber, P., Dahl, J., Hermansson, R., 1997. Study of the phenomena affecting the accuracy of a video-based particle tracking velocimetry technique. Experiment in Fluids 22 (6), 482-488.

Wang, T.T., Lo, W., Ding, Y.C., Liu, S.T., 2007a. Study on tunnel excavation disturbed zone in weak rock with time-dependent behavior. In: Eleventh Congress of the International Society for Rock Mechanics, Lisbon, Portugal.

Wang, T.T., Wang, W.L., Huang, T.H., Lee, C.H., 2005. Tunnel rehabilitation in Taiwan, recently cases study. In: International Symposium on Design, Construction and Operation of Long Tunnels to Celebrate the Breakthrough of the $12.9 \mathrm{~km}$ Hsuehshan Tunnel, Taipei, Taiwan.

Wang, X., Huang, X.F., Bian, F.L., Sun, J., 2007b. 3D triangulation of terrestrial laser scanning data based on spherical projection. In: Proceedings of SPIE - The International Society for Optical Engineering, vol. 6751, Geoinformatics 2007: Cartographic Theory and Models, Nanjing, China. 